\section{Kaugummi fürs Ohr}

Kauen Kinder Kaugummi mit dem Süßstoff Xylit, können sie dadurch jede vierte Otitis media vermeiden. Die bakterienhemmende Eigenschaft von Xylit wirkt sich bis zum Mittelohr aus, ergab eine Cochrane-Analyse von drei placebokontrollierten Studien aus Finnland. In diesen erhielten über 1.800 Kinder unter zwölf

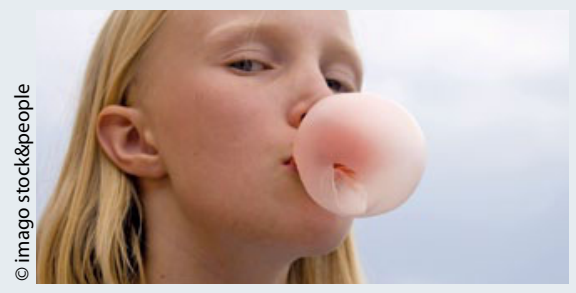

Jahren entweder Kaugummis, Lutschtabletten oder einen Sirup mit dem Süßstoff in der Tagesdosis von 8-10 g oder aber solche Präparate ohne Xylit. Im Schnitt dauerten die Studien zwei bis drei Monate. Insgesamt kam es in dieser Zeit zu 473 Mittelohrentzündungen. Diese waren in den Gruppen mit Xylitgabe deutlich seltener als in der Placebogruppe. Am effektivsten schien der Kaugummi: Die Otitis-media-Rate war um $41 \%$ reduziert, mit Lutschltablette und Sirup dagegen nur um $20 \%$.

Dr. Brigitte Moreano

Azarpazhooh A et al. Cochrane Database Syst Rev 2011 Nov 9;11: CD007095

\section{Phototherapie bei Neugeborenen} lässt Melanozyten wuchern

An 15 eineiigen und 43 zweieiigen Zwillingspaaren zwischen drei und 30 Jahren untersuchten ungarische Forscher, ob ein Zusammenhang zwischen einer Phototherapie in den ersten Lebenstagen und der späteren Anzahl melanozytärer Nävi besteht. Das Besondere an diesen Zwillingspaaren war, dass kurz nach der Geburt nur jeweils ein Geschwister mit blauem kurzwelligem Licht bestrahlt wurde. Die Probanden, die in den ersten Tagen ihres Lebens unter einer Phototherapielampe lagen, hatten signifikant mehr melanozytäre Nävi auf der Haut als diejenigen, die der Bestrahlung nicht ausgesetzt waren. Ähnliches zeigte sich auch am Auge: Die Blaulichttherapie war mit einer deutlich höheren Prävalenz melanozytärer Veränderungen am Auge assoziiert. Die untersuchten Genpolymorphismen hingegen blieben ohne Effekt auf die Anzahl melanozytärer Veränderungen. Dr. Dagmar Kraus

Csoma Z et al. Pediatrics $2011 ; 128$ : e856-64e

\title{
Entlastung des Gesundheitswesens durch Rotavirusimpfung
}

Die Wirksamkeit der Rotavirusimpfung ist zwar mittlerweile sehr gut belegt, die meisten Untersuchungen haben jedoch nur den Rückgang der Hospitalisierungen erfasst und nicht den Einfluss der Impfung auf die Inanspruchnahme des Gesundheitswesens insgesamt. Dieser wurde nun in einer aktuellen Studie untersucht.

ennifer Cortes und Mitarbeiter von den Centers for Disease Control (CDC) in Atlanta, USA, haben in einer jüngst vorgelegten Untersuchung die gesamte Inanspruchnahme des Gesundheitswesens wegen Rotaviruserkrankungen bei Kindern unter fünf Jahren analysiert. Verglichen wurden die Daten vor und nach Einführung der routinemäBigen Rotavirusimpfung in den USA. Dort wurde die Rotavirusimpfung mit der pentavalenten Vakzine (RV5) im Februar 2006 routinemäßig eingeführt. Zuvor wurden in den USA jährlich 400.000 Arztbesuche und 200.000 Besuche in Notfallambulanzen durch Rotavirusinfektionen verzeichnet sowie 55.000 Hospitalisierungen und 20 bis 60 Todesfälle bei Kindern unter fünf Jahren.

In der Periode von Januar bis Juni 2008 und 2009 gingen die Hospitalisierungen wegen Rotavirusinfektionen um $89 \% \mathrm{zu}$ rück verglichen mit dem Zeitraum vor Einführung der Impfung. Es muss dabei bedacht werden, dass auch in den untersuchten Zeitraum nach Einführung der Impfung noch nicht alle Kinder tatsächlich alle drei Impfungen erhalten hatten. Der Rückgang der Notfallambulanzbesuche wegen Durchfalls ist verständlicherweise weniger eindrucksvoll, da in dieser

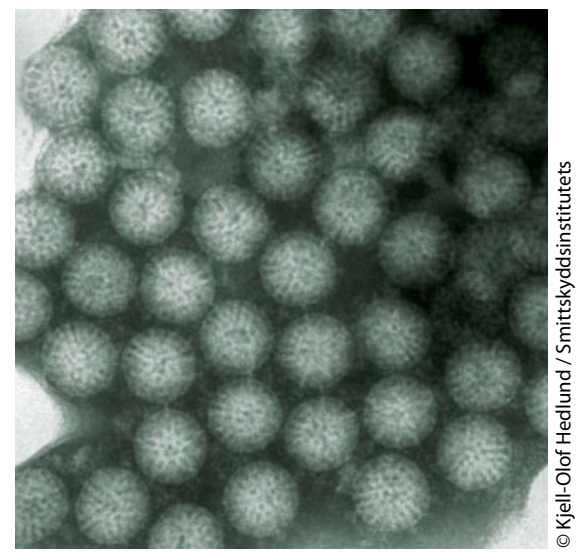

Rotaviren unter dem Mikroskop
Zahl natürlich auch Durchfallerkrankungen, die nicht durch Rotaviren ausgelöst sind, erfasst werden. Immerhin sind in den beiden Beobachtungsperioden jeweils Rückgänge von 37 respektive $48 \%$ zu verzeichnen. Ein indirekter Nutzen der Impfung, das heißt eine positive Auswirkung der routinemäßige Impfung auf nicht geimpfte Kinder war eindeutig in der Beobachtungsperiode Januar bis Juni 2008 nachweisbar, in der entsprechenden Periode 2009 war dieser indirekte Effekt nur bei der ambulanten Versorgung nachweisbar. Allein durch Verminderung der Hospitalisierungszahlen konnten in den USA in den Jahren von 2007 bis 2009278 Millionen US-Dollar an Kosten eingespart werden.

Cortes JE et al. Rotavirus vaccine and health care utilization for diarrhea in U.S. children. N Engl J Med 2011; 365: 1108-17

Kommentar: Es wird zunehmend deutlich, dass die routinemäßige Einführung der Rotavirusimpfung unter Kostengesichtspunkten attraktiv ist. In Deutschland ist die Impfung in drei ostdeutschen Ländern (Mecklenburg-Vorpommern, Sachsen, Thüringen) und in einem westdeutschen Bundesland (Schleswig-Holstein) inzwischen öffentlich empfohlen. Für eine vollständige KostenNutzen-Analyse müssen die Kosten der Impfung und auch die indirekten Kosten durch die Erkrankung des Kleinkindes (z. B. Arbeitsfehlzeiten der Betreuungsperson) noch berücksichtigt werden.

Die Autoren weisen darauf hin, dass selbst eine minimale Erhöhung des Invaginationsrisikos um etwa ein bis zwei Fälle pro 100.000 geimpfte Säuglinge weniger als $2 \%$ der eingesparten Kosten durch eine geringere Hospitalisierungsrate wegen Rotavirusenteritis verursachen würde. Eine solche Erhöhung des Invaginationsrisikos wurde in den USA allerdings nicht beobachtet.

Dr. Hartmut Koch 